\title{
Economic Load Dispatch using Modified Vortex Search Algorithm
}

\author{
P. Suresh, Nihal Baldwa, Shubhi Karn, Rahul Agrawal, Debajoyoti Paladhy, Tarway Preeti \\ Rakesh, Sanjeen Suman
}

\begin{abstract}
This paper presents an efficacy optimization technique for solving Economic Load Dispatch (ELD) problem in power systems. Economic load dispatch has become more complicated with added ramp rate limits constraints to the problem. Latterly their were many traditional approach's applied to ELD problem like Merit order loading, particle swarm optimation technique. This approach can be efficient for ELD problems. It cannot be applied due to high elaboration of their solutions. The Vortex Search Algorithm (VSA) is a newly advanced algorithm influenced by a vortex arrangement which can be designed as a number of nested circles. VSA approach was advanced from state of stirring liquids. The expediency and effectiveness of this approach is determined in different cases. It has no additional problem-specific parameters and it can be applicable to the optimization problems without control parameters tuning. VS Algorithm basically adjusts its step size automatically for the changing values of radius (circles) to improve the solution. In this proposed work, modified vortex search (MVSA) algorithm is applied to solve the ELD problem in some 6-unit test system by considering the system constraints and also the performance of this algorithm can be analyzed in terms of total generation costs and power losses. Obtained results of the test systems will be compared with particle swarm optimization (PSO), Merit order loading, VSA literature. The obtained results will demonstrate the MVSA algorithm is efficient way of solving the ELD problem and finding the output power of all the generation units accurately.
\end{abstract}

\section{INTRODUCTION}

Economic load dispatch is a phenomenon in which the real and reactive power of the generator differs within the limits and consummates the load demand with less fuel cost. It is obligatory to operate the units economically, in the grid system. The impetus of economic load dispatch is to regulate the optimal power generation of the units engaging in

Revised Version Manuscript Received on 10, September 2019.

P. Suresh, Assistant Professor, Department of Electrical And Electronics, SRM Institute of Science And Technology, Kattankulathur, Chennai, Tamilnadu, India.

Nihal Baldwa, UG students, Department of Electrical And Electronics, SRM Institute of Science And Technology, Kattankulathur, Chennai, Tamilnadu, India.

Shubhi Karn, UG students, Department of Electrical And Electronics, SRM Institute of Science And Technology, Kattankulathur, Chennai, Tamilnadu, India.

Rahul Agrawal, UG students, Department of Electrical And Electronics, SRM Institute of Science And Technology, Kattankulathur, Chennai, Tamilnadu, India.

Debajoyoti Paladhy, UG students, Department of Electrical And Electronics, SRM Institute of Science And Technology, Kattankulathur, Chennai, Tamilnadu, India.

Tarway Preeti Rakesh, UG students, Department of Electrical And Electronics, SRM Institute of Science And Technology, Kattankulathur, Chennai, Tamilnadu, India.

Sanjeen Suman, UG students, Department of Electrical And Electronics, SRM Institute of Science And Technology, Kattankulathur, Chennai, Tamilnadu, India. supplying the load. It models the electric power system and ejects the accessible generated resources to supply a given load for each control area in the most economic manner in real-time operation. The sizes of the electric power system are increasing rapidly to converge with the energy requirement. Therefore, the number of power plants are connected in parallel to supply the system load by an assemblage of the power system.The economic scheduling of the generators focusses on assuring to supply load demand to the system all the time by the optimum combination of generators. The economic scheduling problem necessitates two separate steps, which are the online load dispatch and the unit commitment. The unit commitment step focuses on stipulating that unit which will anticipate load of the system over the required period of time at minimum cost. The online load dispatch step dispenses the load among the generating unit. This generating unit is parallel to the system in such a way to reduce the total cost of supplying. It basically fulfils the minute to the minute essentials fof the system. A power system is composed of several power plants and the power plant has several generating units. At any point of time, the composite load in the system is met by the generating units in distinct power plants. Economic load dispatch helps in determining the power output of each power plant as well as of each generating unit .This phenomenon minimizes the overall cost of fuel required to serve the system load.

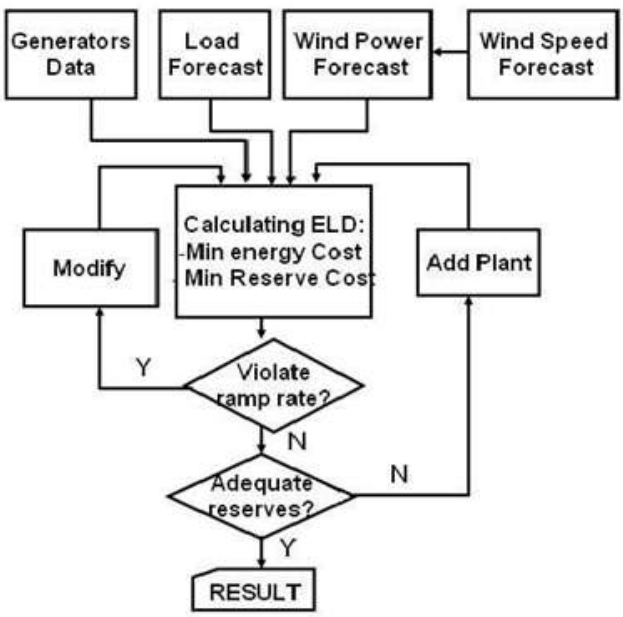

Fig 1 
The outcome of the ELD problem solving are minimizing the cost of total generation, minimizing the losses in the system, increasing efficiency of system, minimizing the voltage varitation. Finding the product of activepower and reactive power produced by the generators so that the total generating cost off the system is minimized traditionally that is known as problem of economic load dispatch (ELD). Most of generating systems in India are of three types: nuclear generating station, hydropower generating station, and thermal generating station (using fossil fuels such as coal, oil and gas). Nuclear generating stationare generally running at constant power levels. In hydropower generating stationunits the operating cost tend not to vary with power levels. The changes in operating cost of thermal power plants are significantly seen with the output levels. In this paper, our objective is to focus on the problem of ELD for power systems consisting of thermal units only as generators. The intent is to decrease the total generating cost by considering the following driving constraints: load factor,margin requirements of the system, economical limits, ramp rate of each unit, restricted operating zones, emission allowance of the system, supporting multiple I/O curves. Constraints are imposed on the model as there is a need to keep a power stability, so that the drift on any line must not be more than its maximum capacity resulting in an kind of damage. The aim of ELD problem is to decrease the system constraints.

CTotal $=\mathrm{f}(\mathrm{PG} 1, \mathrm{PG} 2, \ldots, \mathrm{PGn})$

$\mathrm{CTotal}=$ total generating cost

$\mathrm{PG} 1, \mathrm{PG} 2, \ldots, \mathrm{PG}=$ is separate generation of $\mathrm{n}$-number of units.

Although in this paper we will consider 6 generator units. There are few algorithms to solve the problem of economic load dispatch, which are: Merit order loading, range elimination, vortex algorithm, particle swarm optimization, genetic algorithm, binary selection.

\section{CONSTRAINTS CONSIDERED IN CALCULATION}

In this paper, we are calculate the ELD problem for 6-unit system, by observing the system constraints. Then product of all the considerdmethod is analyzed in terms of total generation cost and power loss's technique is an efficient technique defined to a single solution-based metaheuristic algorithm which evolves solution iteratively. Many different optimization methods have been applied to solve these problems such asmerit order loading, particle swarm optimization and vsa. Main purpose of ELD is that the fuel cost of all the generator should be at base line while generating the total demanded power in an less cost. The constraints that are considered for solving ELD problem using MVSAare-Valve Point Loading Effect, generators limits, power balance, ramp rate limits, prohibited zone.

\section{VALVE POINT LOADING EFFECT}

For more coherent and correct modeling of fuel cost function satisfactory formulations need to be done. The generator units which have multi-valve steam turbines manifest a greater distinction in the fuel-cost functions. In the heat rate curve of the generators a ripple like effect is produced by the multi valve steam turbines during the valve opening process. The importance of this valve point loading effect is that the actual cost curve function of a large steam plant is not continuous and more importantly it is non-linear. These effects are taken into consideration in the ED problem by layering the basic quadratic fuel-cost characteristics with the rectified sinusoid component The basic cost function equation of ELD is given as:

$$
\mathrm{F}_{\mathrm{i}}=\mathrm{a}_{\mathrm{i}}+\mathrm{b}_{\mathrm{i}} \times \mathrm{P}_{\mathrm{i}}+\mathrm{c}_{\mathrm{i}} \times \mathrm{P}
$$

The losses at the power systems have grown due to open stream valves and this effect is called valve point loading effect. Because of this, the total cost value is also increased. The objective function is formed by considering valve point effect and is defined as:

$$
\mathrm{F}_{\mathrm{i}}=\mathrm{a}_{\mathrm{i}}+\mathrm{b}_{\mathrm{i}} \times \mathrm{P}_{\mathrm{i}}+\mathrm{c}_{\mathrm{i}} \times \mathrm{P}_{\mathrm{i}}^{2}+\mathrm{e}_{\mathrm{i}} \times \sin \left(\mathrm{f}_{\mathrm{i}} \times\left(\mathrm{P}_{\mathrm{i} \min }-\mathrm{P}_{\mathrm{i}}\right)\right)
$$

Fi constitutes resulting fuel cost value

Piconstitutes power of thermal generator

ai, bi, ciare cost coefficients

ei , fiare valve point loading coefficients of thermal generator unit $\mathrm{i}$.

The total cost value of system is procured by summing the cost values of every thermal generator units.

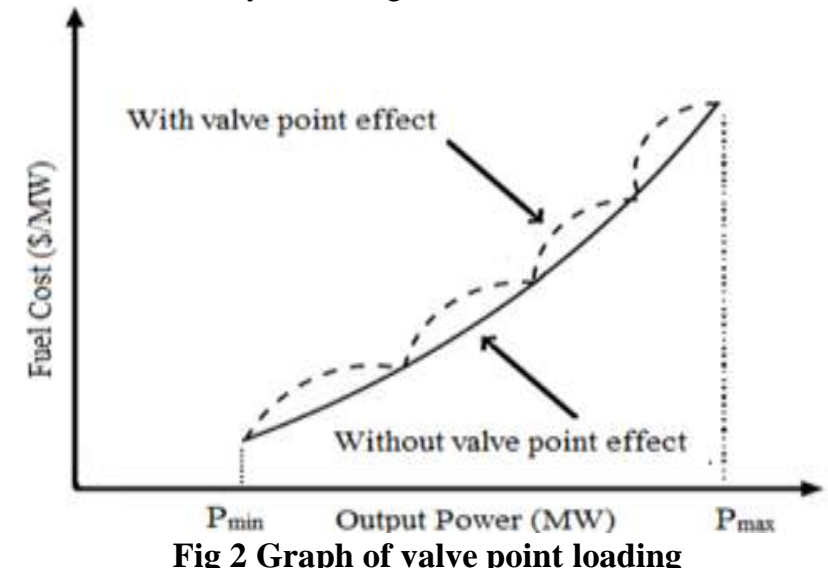

\section{GENERATOR LIMITS}

The voltage of a generator is limited due to 2 reasons : 1 ) Dielectric 2) Heating in core due to excess magnetic flux. However, the maximum continuous limit due to excess flux is lower than that due to dielectric breakdown considerations. Therefore the limit due to excess flux is the "determining" limit. The flux in the core is affected by the frequency ( core flux is proportional to voltage/frequency). Armature Winding (heating) Limit:Heating of armature winding results due to the resistive loss in armature windings.

Field Winding (heating) Limit: Ohmic loss and consequent heating in the field winding, imposes a restriction on the maximum field current. Since field winding current is proportional to the field voltage (after electrical transients have died down), this limit is equivalent to a field voltage limit. Field current is higher when the generator supplies reactive power and is over-excited 
Core-end heating limit: Core-end heating results when field current is low (under-excitation). During underexcitation conditions, the axial flux in the end region is enhanced. This results in heating which may limit the capability of a generator.

Thermal generator units must work between certain range which is maximum and minimum power range and this power range is distinct for distinct units:

$\mathrm{Pi}, \max \geq \mathrm{Pi} \geq \mathrm{Pi}$,min

$\mathrm{Pi}$,min - constitutes lower limit of generating unit i.

$\mathrm{Pi}$,max - constitutes upper limit of generating of unit i.

\section{POWER BALANCE}

The total produced power at the thermal power plants should meet the demand power by consumers. That is why, transmission line losses should be considered. The total generated power procured is the sum of total demand power and total transmission line losses.

Pgi $=$ PD + Ploss

PGi - gross Generated Power

PD - gross Demand Power

Ploss - gross Transmission loss

Transmission line losses are calculated as follows:

$$
\mathrm{P}_{\text {loss }}=\Sigma \Sigma \mathrm{N}_{\mathrm{j}=1} \mathrm{~N}_{\mathrm{i}=1} \mathrm{P}_{\mathrm{i}} \mathrm{B}_{\mathrm{ij}} \mathrm{P}_{\mathrm{j}}+\sum \mathrm{N}_{\mathrm{i}=1} \mathrm{~B}_{0 \mathrm{i}} \mathrm{P}_{\mathrm{i}}+\mathrm{B}_{00}(3)
$$

$\mathrm{N}$-constitutes total thermal power plant

$\mathrm{Bij}, \mathrm{B} 0 \mathrm{i}$ and B00 are transmission loss coefficients

\section{RAMP RATE LIMITS}

When a thermal generator unit is working at a certain point, the working point can be increased to a certain level that is determined by the up-ramp rate limit or decreased to a certain level determined by the down ramp rate limit. This condition are-

Max (Pi min, Pi0 - Dri $\leq \mathrm{Pi} \leq \min (\mathrm{Pi} \max , \mathrm{Pi} 0+\mathrm{URi})$

URi -ramping up limit

DRi- ramping down limit

Pi0 - Precursory produced power

Pi- produced Power of Unit i

\section{5.) PROHIBITED ZONE}

In some particular cases, thermal generator units do not work due to mechanical corruption in some specific power range.

These conditions can be expressed as follows:

$\mathrm{Pi} \min \leq \mathrm{Pi} \leq \mathrm{Pi}, 1$ lower bound

$\mathrm{Pi}, \mathrm{j}-1$ upper bound $\leq \mathrm{Pi} \leq \mathrm{Pj}$ lower bound

$\mathrm{Pi}$,ni upper bound $\leq \mathrm{Pi} \leq \mathrm{Pi} \max$

$\mathrm{j}$ constitutes number of prohibited zones of unit $\mathrm{i}, \mathrm{j}=2,3,4$ ...ni

Pj lower boundconstitutesjth prohibited zone oflower limit

$\mathrm{Pj}$ upper boundconstitutesjth prohibited zone of upper limit

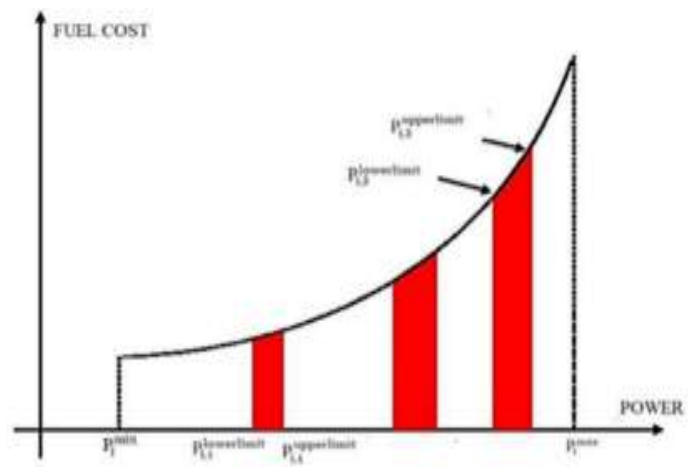

Fig 3 Graph for prohibited zone

\section{VORTEX SEARCH ALGORITHM (VSA)}

The VSA is a newly developed metaheuristic algorithm in which the vortex pattern that can be modeled as a number of nested circles together. When considered in two dimensional spaces, vortex patterns are likely to be taken as nested circles. The biggest of these nested circles is beginning circle and it can be defined as the first search space. This algorithm basically adjusts step sizes automatically according to change in value of the radius of circles to improve solutions. In this method, we calculate theELD problem for 6-units, taking the system constraints. Then product of this algorithm is observed in terms of total cost for generation and power loss. It is an efficient technique that is used to define a single solution-based metaheuristic algorithm that evolves solution iteratively. Many different optimization methods have been applied to solve these problems such as merit order loading, particle swarm optimization and vsa.. Main aim of ELD is that the total fuel cost of all the generator should we at base level and the total demanded power should be produced in an efficient way. The constraints that are considered:

VSA are -Valve Point Loading Effect, generators limits, power balance, ramp rate limits, prohibited zone.

VSA is calculated as follows:

\section{GENERATING THE INITIAL SOLUTIONS}

VS Algorithm generates initial solution by modeling the no. of nested circles. For 2-dimensional optimization problems initial center is found at -:

$$
\mu 0=(\text { upper limit }+ \text { lower limit }) / 2(4)
$$

where the upper and lower limits are maximum and minimum power generated by the unit respectively. 


\section{ECONOMIC LOAD DISPATCH USING MODIFIED VORTEX SEARCH ALGORITHM}

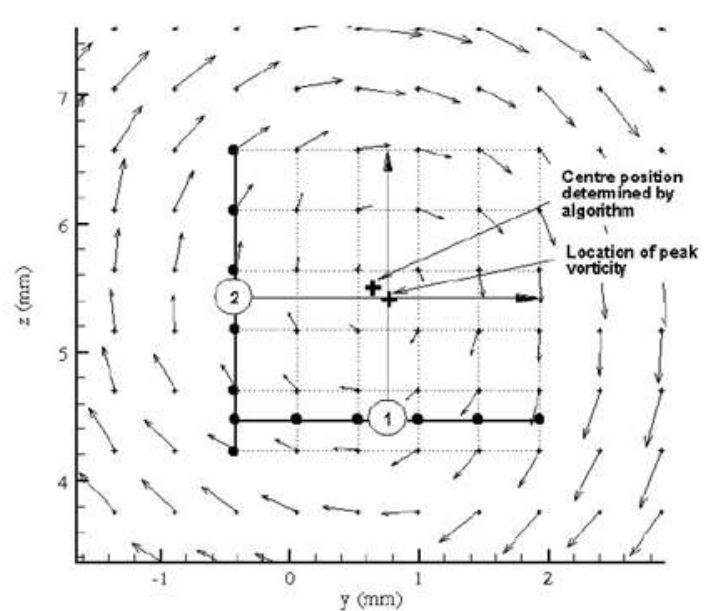

Fig 4 Vortex pattern

\section{GENERATING THE CANDIDATE SOLUTIONS}

By using Gaussian distribution, a number of neighbor solutions $\mathrm{Ct}(\mathrm{S})$ are created randomly in 2-dimensional search space, where $t$ is the iteration index which is selected as 0 at the beginning of the optimization process. The value of $\Sigma$, which represents a covariance matrix, is calculated by using

$$
\Sigma=\sigma^{2}[\mathrm{I}] \quad(5)
$$

$\sigma 2=$ variance

$\mathrm{I}=\mathrm{dxdidentity} \mathrm{matrix.}$

The value of the initial standard deviation can be defined as the:

$$
\sigma 0=[\max (\text { upperlimit })-\min (\text { lowerlimit })] / 2(6)
$$

The initial solution is calculated by the generated candidate solution. The candidate solution obtained must be in the range of upper and lower limit of the system. The generated candidate solution which are not in range are shifted into that range with help of equation given by

$$
\mathrm{s}_{\mathrm{i}}^{\mathrm{k}}=\text { lower limit }{ }^{\mathrm{i}}+\left(\text { upper limit }- \text { lower limit }{ }^{\mathrm{i}}\right) \times \text { rand }
$$

After doing certain iterations, the best solution is chosen. That best solution is termed as ' $\mathrm{s}$ '. This solution is considered and recent center $\mathrm{u} 0$ is repositioned to $\mathrm{s}$. New candidate solution will give the new center. After getting all candidate solutions, the results are correlated with all previous best solution. After correlation, if it is a better solution then that solution is designated as best solution. This process takes place in every step of iteration. After every iteration, radius of the circles must be decremented. Because of this reason, a new term called as the inverse gamma function (gammaincinv) is used and new radius is found for every iteration as follows:

$$
r t=\sigma \times \frac{1}{x} \times \gamma \text { in } \times \operatorname{cin} \times \operatorname{gamma}(x, a t)
$$

at=variable parameter and it dependent a0and $t$ and MaxItr. This function shown as follows:

$$
\mathrm{a}_{\mathrm{t}}=\mathrm{a}_{0}-\left(\mathrm{t} / \operatorname{Max}_{\mathrm{Itr}}\right)
$$

Where, t constitutes iteration number,

MaxIt constitutes maximum number of iteration.

In order to cover all the search space, a0 is chosen as 1 .

\section{STEPS INVOLVED IN CALCULATING VSA}

This section summarizes the optimization processes of the VS based ELD problem, as follows:

Step 1) Define the boundary values of the 6-unit system, lower and upper boundaries of the units, maximum number of iterations and number of candidate solutions.

Step2) Generate initial solutions representing a potential solution of the 6-unit test system mentioned .Eqn(4)

Step3) Check all the constraints. Any violated variable of $\mathrm{C}(\mathrm{S})$ is directly set to given limit to satisfy the inequality constraints of the units. For the equality constraints, if any variable of $\mathrm{Ct}(\mathrm{S})$ exceeds the constraints, add the term to the main function.

Step 4) Calculate total generation costs of the units.

Step5) Improve the candidate solutions of $\mathrm{Ct}(\mathrm{S})$ by decreasing the radius $\mathrm{rt}$ according.

Step 6) Memorize the best solution found so far.

Step7) If the iteration has reached the maximum number of iterations, stop the optimization process; otherwise, increase the iteration by one and go to Step 3.

\section{MODIFIED VORTEX SEARCH ALGORITHM}

The objective of this work is to reduce the number of iteration for the calculation that has been done in VSA. The results will be the same but the effort in calculating result is reduced. In this paper 6-systems are considered.

\section{GENERATING THE INITIAL SOLUTIONS}

VS Algorithm generates initial solution by modeling the no. of nested circles. For 2-dimensional optimization problems initial center is found at -:

$\mu=$ (upper limit + lower limit) $/ 2$

$\mu=$ (upper limit - lower limit) $/ 2$

where the upper and lower limits are maximum and minimum power generated by the unit respectively.

\section{GENERATING THE CANDIDATE SOLUTIONS}

By using Gaussian distribution, a number of neighbor solutions $\mathrm{Ct}(\mathrm{S})$ are created randomly in 2-dimensional search space, where $t$ is the iteration index which is selected as 0 at the beginning of the optimization process. The value of $\Sigma$, which represents a covariance matrix, is calculated by using

$$
\Sigma=\sigma 2[\mathrm{I}]
$$

$\sigma 2=$ variance

$\mathrm{I}=\mathrm{dxd}$ identity matrix.

The value of the initial standard deviation can be defined as the:

$\sigma 0=[\max ($ upperlimit $)-\min ($ lowerlimit $)] / 2(13)$ 
The initial solution is calculated by the generated candidate solution. The candidate solution obtained must be in the range of upper and lower limit of the system. The generated candidate solution which are not in range are shifted into that range with help of equation given by

$$
\mathrm{s}_{\mathrm{i}}^{\mathrm{k}}=\text { lower limit }{ }^{\mathrm{i}}+\text { (upper limit - lower limit }{ }^{\mathrm{i}} \text { ) } \times \text { rand }
$$

after doing certain iterations, the best solution is chosen. And it is termed as ' $\mathrm{s}$ '. This solution is considered and recent center $\mathrm{u} 0$ is repositioned to $\mathrm{s}$. Now the new candidate solution will give the new center. After getting all candidate solutions, a comparison is made with the results of all previous best solutions. After comparison, if it is occurs as the better solution then that solution is designated as the best solution. This process proceeds in every step of iteration. After every iteration, radius of the circles must be decremented. For this reason, a new term known as inverse gamma function (gammaincinv) is used and new radius is found for every iteration by using the formula :

$$
r t=\sigma \times \frac{1}{x} \times \gamma \text { in } \times \operatorname{cin} \times \operatorname{gamma}(x, a t)(15)
$$

at constitutes variable value and it is dependent on $\mathrm{a} 0$ and $\mathrm{t}$ and MaxItr.

The function shown follows as:

at $=\mathrm{a} 0-(\mathrm{t} /$ Maxitr $)$

Where,

t constitutes iteration number,

MaxItr constitutes maximum number of iteration.

In order to cover all the search space $\mathrm{a} 0$ is chosen

\section{STEPS TO SOLVE ELD PROBLEM USING MVSA \& RESULTS}

This section summarizes the optimization processes of the VS based ELD problem, as follows:

Step 1) Define the boundary values of the 6-unit system, lower and upper boundaries of the units,, maximum number of iterations and number of candidate solutions.

Step2) Generate initial solutions representing a potential solution of the 6-unit test system mentioned. Equ(10) and equ(11)

Step3) Check all the constraints. Any violated variable of $\mathrm{C}(\mathrm{S})$ is directly set to given limit to satisfy the inequality constraints of the units. For the equality constraints, if any variable of $\mathrm{Ct}(\mathrm{S})$ exceeds the constraints, add the term to the main function.

Step 4) Calculate the total generation costs of the units.

Step5) Improve the candidate solutions of $\mathrm{Ct}(\mathrm{S})$ by decreasing the radius $\mathrm{rt}$ according.

Step 6) Memorize the best solution found so far.

Step7) If the iteration has reached the maximum number of iterations, stop the optimization process; if not increment the valve of iteration and go back to Step 3.

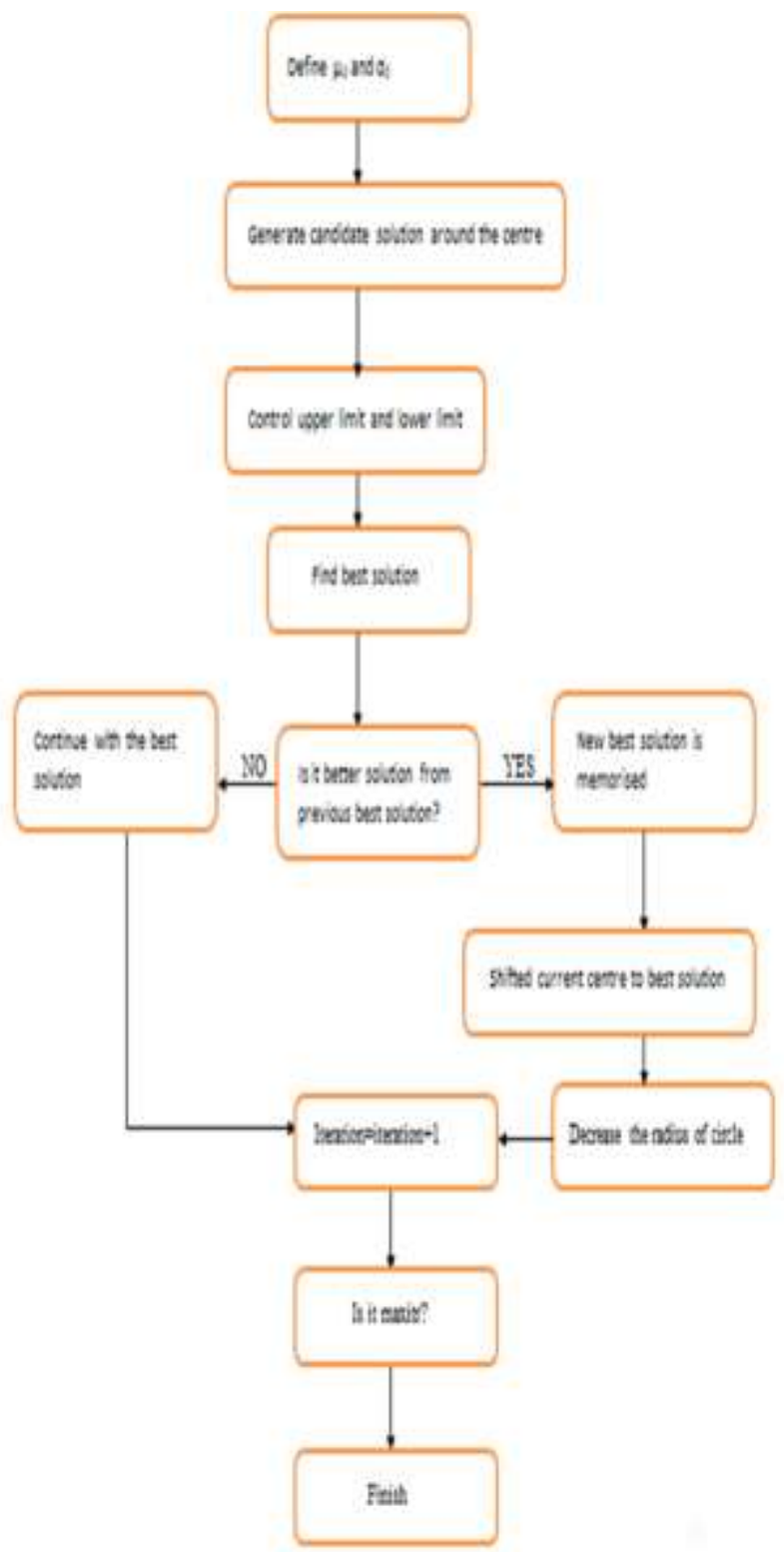

Fig 5 Flow chart for VSA

\section{NUMERICAL RESULTS OF EXISTING METHODS}

Taking 6 Cases and for each case transmission line loss coefficient are same and given as follows

$\mathrm{B}=10-4 \mathrm{x}$

Table1

\begin{tabular}{|l|l|l|l|l|l|}
\hline 0.17 & 0.12 & 0.07 & -0.01 & -0.05 & -0.02 \\
\hline 0.12 & 0.14 & 0.09 & 0.01 & -0.06 & -0.01 \\
\hline 0.07 & 0.09 & 0.31 & 0.01 & -0.10 & -0.06 \\
\hline-0.01 & 0.01 & 0.00 & 0.24 & -0.06 & -0.08 \\
\hline-0.05 & -0.06 & -0.10 & -0.06 & 1.29 & -0.02 \\
\hline-0.02 & -0.01 & -0.06 & -0.08 & -0.02 & 1.5 \\
\hline
\end{tabular}

$\mathrm{B} 0=10-4 \times$

$\begin{array}{llllll}-3.9080 & -1.2970 & 7.0470 & 0.5910 & 2.1610 & -6.6350\end{array}$ 


\section{Case for ELD with Ramp Rate}

In this case two distinct constraints, that are ramp rate limits and transmission line losses, are pertained to the system. The data for cost coefficients, ramp rate limits and maximum and minimum limits of generator are given below. Results of MVSA calculated with different optimization techniques

Table2

\begin{tabular}{|l|l|l|l|l|l|l|l|l|}
\hline Unit & $\mathrm{a}$ & $\mathrm{b}$ & $\mathrm{c}$ & Po & U R & DR & Pmin & Pmax \\
\hline 1 & 240 & 7 & 0.0070 & 440 & 80 & 120 & 100 & 500 \\
\hline 2 & 200 & 10 & 0.0095 & 170 & 50 & 90 & 50 & 200 \\
\hline 3 & 220 & 8.5 & 0.0090 & 200 & 65 & 100 & 80 & 300 \\
\hline 4 & 200 & 11 & 0.0090 & 150 & 50 & 90 & 50 & 150 \\
\hline 5 & 220 & 10.5 & 0.0080 & 190 & 50 & 90 & 50 & 200 \\
\hline 6 & 190 & 12 & 0.0075 & 150 & 50 & 90 & 50 & 120 \\
\hline
\end{tabular}

Table 3

\begin{tabular}{|l|l|l|l|}
\hline P(MW) & VSA & PSO & MOL \\
\hline P1 & 456.063 & 494.24 & 453.39 \\
\hline P2 & 173.3751 & 115.63 & 165.279 \\
\hline P3 & 265.39 & 264.41 & 265.223 \\
\hline P4 & 142.4373 & 140.71 & 124.21 \\
\hline P5 & 165.0545 & 180.69 & 168.22 \\
\hline P6 & 77.1690 & 85.83 & 121.00 \\
\hline PLOSS & 13.4889 & 13.22 & 13.24 \\
\hline COST & 15449 & 15490 & 15454.13 \\
\hline
\end{tabular}

Table4

\begin{tabular}{|l|l|l|l|}
\hline P(MW) & MVSA & PSO & MOL \\
\hline P1 & 456.063 & 494.24 & 453.39 \\
\hline P2 & 173.3751 & 115.63 & 165.279 \\
\hline P3 & 265.39 & 264.41 & 265.223 \\
\hline P4 & 142.4373 & 140.71 & 124.21 \\
\hline P5 & 165.0545 & 180.69 & 168.22 \\
\hline P6 & 77.1690 & 85.83 & 121.00 \\
\hline PLOSS & 13.4889 & 13.22 & 13.24 \\
\hline COST & 15449 & 15490 & 15454.13 \\
\hline
\end{tabular}

\section{RESULT}

As in table 3 the calculations we got for VSA and other methods. VSA is more effective in the calculations and effectiveness.

$\mathrm{VSA}=15448$

$\mathrm{PSO}=15489$

$\mathrm{CPSO}=15481.8$

MOL $=15453.1$

Results for table 3

When MVSA is correlated with PSO, CPSO and MOL techniques from the table 4,it is quite evident that from the Table 4 proposed MVSA method is capable of finding the best solutions at minimum cost value with reduced iterations.

As we are comparing MVSA and VSA

1. the number of iterations is less in MVSA

2. Easy to implement

3. Effort is less

4. Result is same as VSA

\section{CONCLUSION}

When modified vortex search algorithm is compared with other methods such as particle swarm optimization, genetic algorithm and merit order loading it was found that

Feasibility and effectivity. Other techniques like particle swarm optimization, genetic algorithm etc uses costumer-defined values. If the costumer fails of impart correct values this method will not work efficiently. In current studies the VSA has been proven more beneficial in solving the real world problem. As we can say that the MVSA will also impart the same functions in real time problems.

The simulation results of PSO have been compared to the different algorithms used before in literature and the obtained results demonstrate that MVSA is capable of solving the ELD minimization problem efficiently and finding the output power of all the generation units properly. The VS algorithm preforms better than the other competitors in terms of minimizing total generation cost. It also provides a good balance between exploration and exploitation that result in high accuracy when searching for the solutions. In future, the VS algorithm may be used for solutions of other power system optimization problems such as dynamic economic dispatch, generation scheduling and reactive power flow. As seen in the given results the MVSA as less number of iterations it is more simple, less time consuming for the user to calculated the ELD problem. Hence it is concluded that MVSA is much more efficient. Then VSA.

\section{REFERENCE}

\section{STEPS TO SOLVE ELD PROBLEM USING MVSA}

1. K. Dasgupta, S. Baneljee, C. K., "An Analysis of Economic Load Dispatch with Ramp-rate limit constraints using Different Algorithms", Int. Con. Ind. Inst. Control, pp. 232-236,2015.

2. T. Sen. H. D. Mathur, "A New Approach To Solve Economic Dispatch Problem Using A Hybrid ACOABC- HS Optimization Algorithm", Electrical Power and Energy Systems, vol. 78, pp. 735- 744, 2016.

3. A. Farag, S. AI-Baiyat, T. C. Cheng, "Economic load dispatch multi objective optimization procedures using linear progratmning techniques" IEEE Trans. Power Syst.,vol. 10, pp. 731-738, 1995.

4. P. Bhui, N. Senroy, "A Unified Method for Economic Dispatch with Valve Point Effects", IEEE Int. Con. Power Systems, pp. 1-5,2016.

5. P. Sriyanyong, "An Enhanced Particle Swarm Optimization For Dynamic Economic Dispatch Problem Considering Valve-Point Loading," Proc. the Forth 1ASTED International Conference on Power and Energy Systems (AsiaPES 2008), pp. 167-172,2008.

6. C. L. Chiang, "Genetic-based algorithm for power economic load dispatch" LET Gener. Trans. Distrib., vol. I , pp. 261-269, 2007.

7. S. Hemamalini, S. P. Simon, "Artificial bee colony algorithm for economic load dispatch problem with non-smooth cost functions" Electr. Power Compo. Syst., vol. 38, pp. 786-803,2010.

8. B. Dogan, T. Olmez, "A New Metaheuristic For Numerical Function Optimization: Vortex Search Algorithm", Information Sciences, vol. 293, pp. 125-145, 2015.

9. B. Dogan B., T. Ohnez, "Vortex Search Algorithm For The Analog Active Filter Component Selection Problem", International Journal of Electronics and Comrninications, vol. 69, pp. 1243-1253, 2015. 
10. M. Saka, I. Eke, S. S. Tezcan, M. C. TaplamaclOglu, "Application of VOItex Search Algoritlun to Economic Load Dispatch Including Transmission Losses with Valve-Point Loading Effect", 1st International MeditelTanean Science and Engineering Congress, pp. 2854-2859, 2016.

11. N. Singh, Y. Kumar, "Economic Load Dispatch Using MRPSO with Generator Ramp Rate Limits Constraint", Int. Con. Comp. Int. and Corn. Networks, pp. 632-637, 2012.

12. M. Saka, I. EKE, S. S. Tezcan, M. C. Taplamacioglu., "Economic Load Dispatch Using Vortex Search Algorithm" in 4th International Conference on Electrical and Electronics Engineering, Ankara Turkey, 2017.

13. T. Sen. H. D. Mathur, "A New Approach to Solve Economic Dispatch Problem Using A Hybrid ACOABC-HS Optimization Algorithm", Electrical Power and Energy Systems, 78, 735-744, 2016.

14. L. S. Coelho, V. C. Mariani, "Combining of Chaotic Differential Evolution and Quadratic Programming for Economic Dispatch Optimization with Valve-Point Effect”. IEEE Trans. Power Syst., 21, 989-996, 2006.

15. A. Farag, S. Al-Baiyat, T. C. Cheng, "Economic Load Dispatch Multi Objective Optimization Procedures Using Linear Programming Techniques" IEEE Trans. Power Syst., 10, 731-738, 1995.

16. L. Papageorgiou, E. Fraga, "A Mixed Integer Quadratic Programming Formulation for the Economic Dispatch of Generators with Prohibited Operating Zones" Electr. Power Syst. Res., 77, 1292-1296, 2007.

17. P. Sriyanyong, "An Enhanced Particle Swarm Optimization for Dynamic Economic Dispatch Problem Considering Valve-Point Loading," in Proc. the Forth IASTED International Conference on Power and Energy Systems (AsiaPES 2008), 167-172, 2008.

18. C. L. Chiang, "Genetic-Based Algorithm for Power Economic Load Dispatch” IET Gener. Trans. Distrib., 1, 261-269, 2007.

19. X. S. Yang, S. S. S. Hosseini, A. H. Gandomi, "Firefly Algorithm for Solving Non-Convex Economic Dispatch Problems with Valve Loading Effect", Appl. Soft Comput., 12, 1180-1186, 2012.

20. S. Pothiya, I. Ngamroo, W. Kongprawechnon, “Ant Colony Optimisation for Economic Dispatch Problem with Non-Smooth Cost Function. 